\title{
Characterizing Uniformly Ultimately Bounded Switching Signals for Uncertain Switched Linear Systems
}

\author{
Hai Lin and Panos J. Antsaklis
}

\begin{abstract}
In this paper, the uniformly ultimately bounded (UUB) switching control problem is investigated for a class of continuous-time switched linear systems with parametric uncertainties and exterior disturbances. It is assumed that each subsystem is UUB. First, a class of switching signals, which may contains infinite number of switching and preserves the UUB of the switched systems, is characterized. Then, a switching law is synthesized to improve the disturbance attenuation properties in the sense that all state trajectories can converge into a smaller region than any single subsystem acts alone. The switching law is given as a static state feedback form, and provides a conic partition of the state space. To avoid unstable sliding motions, some modifications are introduced later. The techniques are based on multiple polyhedral Lyapunov functions.
\end{abstract}

\section{INTRODUCTION}

A switched system is a dynamical system that consists of a finite number of subsystems described by differential or difference equations and a logical rule that orchestrates switching between these subsystems. Properties of this type of model have been studied for the past fifty years to consider engineering systems that contain relays and/or hysteresis. Due to its success in application and importance in theory, the last decade has seen increasing research activities in stability [15], [18], [7], controllability [24], [23], observability [1], [9], [12], stabilization [13], [20], [22], [17], and switching optimal control [2], [25] of switched systems.

However, the literature on robust performance of switched systems is still relatively sparse, and most existing results assume that the disturbances are constrained to have finite energy, i.e., bounded $\mathcal{L}_{2}$ norm, see e.g. [10], [29]. In practice, there are disturbances that do not satisfy this condition and act more or less continuously over time. Such disturbances are called persistent, and cannot be treated in the above framework [6]. In this paper, the disturbance attenuation property is in the signal's magnitude sense, i.e., time domain specifications. Moreover, we explicitly consider dynamic uncertainty in the switched system model. Dynamics uncertainty in the plant model is one of the main challenges in control theory, and it is of practical importance to deal with dynamical uncertainties explicitly.

This paper aims to investigate the persistent disturbance attenuation properties in the sense of uniformly ultimate boundedness for a class of switched linear systems with parametric uncertainties and exterior disturbances. It is known

The financial support form Singapore Ministry of Education's AcRF Tier 1 funding is gratefully acknowledged.

H. Lin is with the ECE Dept., National University of Singapore, Singapore 117576. E-mail: elelh@nus.edu.sg

P.J. Antsaklis is with the the EE. Dept., University of Notre Dame, Notre Dame, IN 46556, USA. E-mail: ant saklis.1@nd.edu that even when all subsystems are exponential stable, the switched system may still exhibit unbounded behaviors under some switching signals [15], [7]. Therefore, the first task here is to characterize a class of switching signals, which is called admissible switching, such that the boundedness of the switched systems is preserved. To make the problem nontrivial, the class of admissible switching signals should be large enough to include some interesting cases, such as infinite number of switching and arbitrarily fast switching etc. Next, as one of main motivations to study switched systems, switching among multiple controllers can achieve better performance than each single controller does. Hence, the second task here is to identify a subclass of admissible switching signals such that the switched system has a better disturbance attenuation property. The improvement is in the sense that all trajectories of the switched system would converge into a smaller neighborhood region of the origin than any single subsystem acts alone. The arguments here are mainly based on multiple polyhedral Lyapunov functions. Similar techniques have been used in [14] to design stabilizing switching signals for switched LTI systems. Some preliminary results for the UUB control of discrete-time uncertain switched linear systems appeared in [16].

The rest of the paper is organized as follows. In Section II, the uncertain switched linear system and its persistent disturbance attenuation problems are formulated. In Section III, some preliminary results on polyhedral Lyapunov functions and notations are reviewed. In Section IV, the class of admissible switching signals is characterized. In Section V, the improved performance switching signals are synthesized. The switching law is given as a static state feedback form, and provides a conic partition of the state space. The possible occurrence of sling motions could cost stability, therefore sliding motions are explicitly dealt with in Section VI. It is shown that a simple modification can be made to avoid undesirable sliding motions while guarantee UUB. Finally, conclusions are given.

Notation: The letters $\mathcal{D}, \mathcal{P}, \mathcal{S} \cdots$ denote sets, $\operatorname{int}(\mathcal{P})$ the interior of set $\mathcal{P}$, and $\partial \mathcal{P}$ its boundary. For any real $\lambda \geq 0$, the set $\lambda \mathcal{S}$ is defined as $\{x=\lambda y, y \in \mathcal{S}\}$. A polytope (bounded polyhedral set) $\mathcal{P}$ will be presented either by a set of linear inequalities $\mathcal{P}=\left\{x: f_{i} x \leq g_{i}, i=1, \cdots, s\right\}$, or by the dual representation in terms of the convex hull of its vertex set $\left\{v_{j}\right\}$, denoted by $\operatorname{Conv}\left\{v_{j}\right\}$.

\section{PRoblem Formulation}

Consider a collection of continuous-time linear systems described by the perturbed differential equations with para- 
metric uncertainties

$$
\dot{x}(t)=A_{q}(w) x(t)+E_{q} d(t), \quad t \in \mathbb{R}^{+},
$$

where $q \in Q=\left\{q_{1}, q_{2}, \cdots, q_{N}\right\}$, the state variable $x \in \mathbb{R}^{n}$, and the disturbance input $d \in \mathcal{D} \subset \mathbb{R}^{r}$. Assume that $\mathcal{D}$ is a $\mathrm{C}$-set. The term $\mathrm{C}$-set stands for a convex and compact set containing the origin in its interior. The entries of the state matrix $A_{q}(w): \mathcal{W} \rightarrow \mathbb{R}^{n \times n}$ are assumed to be continuous functions of $w \in \mathcal{W}$, where $\mathcal{W}$ is a given compact set in $\mathbb{R}^{v}$. The parametric uncertainty $w$ is time-variant, and with unknown dependence on time $t$. Without loss of generality, the matrix $E_{q} \in \mathbb{R}^{n \times r}$ is assumed to be a constant matrix.

Combine the family of continuous-time uncertain linear systems (1) with a class of maps, $\sigma: \mathbb{R}^{+} \rightarrow Q$. Then we can define the following time-varying system as a continuoustime switched linear system

$$
\dot{x}(t)=A_{\sigma(t)}(w) x(t)+E_{\sigma(t)} d(t), \quad t \in \mathbb{R}^{+},
$$

where $\sigma$ is called a switching signal.

Because of parameter variations and exterior disturbances, it is only reasonable to expect that the trajectories of the switched system (2) converge into a neighborhood region of the equilibrium (the origin here), as defined below.

Definition 1: The uncertain switched system (2) under switching signal $\sigma(t)$ is Uniformly Ultimately Bounded $(U U B)$ if there exists a $\mathrm{C}$-set $\mathcal{S}$ such that for every initial condition $x(0)=x_{0}$, there exists a finite $T\left(x_{0}\right)$, and $x(t) \in$ $\mathcal{S}$ for $t \geq T\left(x_{0}\right)$.

The above UUB definition is also called practical stability or uniformly ultimate boundedness in the literature. For example, practical stability and stabilization for switched systems were extensively investigated recently in [26], [27]. This paper will mainly focus on the disturbance attenuation properties of switched systems in the sense of the uniformly ultimate boundedness. Given a collection of switching signals, if the switched system (2) is UUB for all these switching signals, then the switched system (2) is said to have finite disturbance attenuation level under this class of switching signals. Throughout the paper, it is assumed that each subsystem (1) is UUB and converges to a C-set $\mathcal{S}_{q}$ respectively, i.e., $\sigma(t)=q$ (for all $t$ ) in Definition 1. It is known that even when all the subsystems are UUB, the switched system (2) could have infinite disturbance attenuation level under certain classes of switching signals. Hence, the first problem being addressed here is to characterize a useful subclass of switching signals under which the switched system (2) remains UUB:

Problem 1: Given the switched system (2), characterize a nontrivial class of switching signals under which the switched system remains UUB.

Here the non-trivialness means that we would like to include the cases that there may be infinite number of switching in $\sigma(t)$ and that arbitrarily fast switching is also possible. An answer for this question is proposed in Section IV based on multiple polyhedral Lyapunov functions. Hence, we first give a short review of some basics for polyhedral Lyapunov functions.

\section{POLYHEDRAL LYAPUNOV FUNCTION}

Following [3], we call a function $\Psi: \mathbb{R}^{n} \rightarrow \mathbb{R}$ a gauge function if $\Psi(x) \geq 0, \Psi(x)=0 \Leftrightarrow x=0$; for $\mu>0$, $\Psi(\mu x)=\mu \Psi(x)$; and $\Psi(x+y) \leq \Psi(x)+\Psi(y), \forall x, y \in \mathbb{R}^{n}$. A gauge function is convex and it defines a distance of $x$ from the origin which is linear in any direction. If $\Psi$ is a gauge function, we define the closed set (possibly empty) $\bar{N}[\Psi, \xi]=\left\{x \in \mathbb{R}^{n}: \Psi(x) \leq \xi\right\}$. It is easy to show that the set $\bar{N}[\Psi, \xi]$ is a C-set for all $\xi>0$. On the other hand, any C-set $\mathcal{S}$ induces a gauge function $\Psi_{\mathcal{S}}(x)$ (Known as the Minkowski function of $\mathcal{S}$ ), which is defined as $\Psi(x) \doteq \inf \{\mu>0: x \in \mu \mathcal{S}\}$. Therefore a C-set $\mathcal{S}$ can be thought of as the unit ball, $\mathcal{S}=\bar{N}[\Psi, 1]$, of a gauge function $\Psi$, and $x \in \mathcal{S} \Leftrightarrow \Psi(x) \leq 1$.

Due to the assumption that each subsystem

$$
\dot{x}(t)=A_{q}(w) x(t)+E_{q} d(t)
$$

is UUB with respect to $\mathcal{S}_{q}$, let us define a Lyapunov function outside $\mathcal{S}_{q}$ for (1) in the following sense [4].

Definition 2: Given a C-set $\mathcal{S}_{q}$, a Lyapunov function outside $\mathcal{S}_{q}$ for the continuous-time system (1) is defined as a continuous function $\Psi_{q}: \mathbb{R}^{n} \rightarrow \mathbb{R}^{+}$such that $\bar{N}\left[\Psi_{q}, \kappa\right] \subseteq$ $\mathcal{S}_{q}$ for some positive scalar $\kappa$, for which the following condition holds: if $x \notin \bar{N}\left[\Psi_{q}, \kappa\right]$ then $\exists \beta_{q}>0$ such that $\mathcal{D}^{+} \Psi_{q}(x(t)) \leq-\beta_{q} \Psi_{q}(x(t))$.

Here $\mathcal{D}^{+} \Psi_{q}(x(t))$ stands for the upper right Dini derivative of $\Psi_{q}(x(t))$ along the trajectories of system (1), which is defined as

$$
\mathcal{D}^{+} \Psi_{q}(x(t))=\lim \sup _{\tau \rightarrow 0^{+}} \frac{\Psi_{q}(x(t+\tau))-\Psi_{q}(x(t))}{\tau} .
$$

Under the assumption that $d(t)$ and $w(t)$ are continuous, then the value of the Dini derivative of the point $x(t)=x$ equals

$\mathcal{D}^{+} \Psi_{q}(x(t))=\lim \sup _{\tau \rightarrow 0^{+}} \frac{\Psi_{q}\left(x+\tau\left[A_{q}(w) x+E_{q} d\right]\right)-\Psi_{q}(x)}{\tau}$, where $x(t)=x, d(t)=d$ and $w(t)=w$ [4].

Based on differential inequality theory, it can be derived from the above Lyapunov function definition that

$$
\Psi_{q}(x(t)) \leq \max \left\{e^{-\beta_{q}\left(t-t_{0}\right)} \Psi_{q}\left(x\left(t_{0}\right)\right), \kappa\right\}, \quad \forall t>t_{0}
$$

for a trajectory $x(t)$ of (1) starting from $x\left(t_{0}\right)$ at time $t_{0}$. This further implies the following result.

Lemma 1: [4] If there exists a Lyapunov function outside $\mathcal{S}$ for the subsystem (1), then it is uniformly ultimately bounded (UUB) in $\mathcal{S}$.

On the other hand, it can be shown that there always exists a polyhedral Lyapunov function for a UUB subsystem (1) in the sense of Definition 2. Therefore, without loss of generality, we assume that each subsystem (1) has a polyhedral Lyapunov function $\Psi_{q}$ outside $\mathcal{S}_{q}$, and that $\bar{N}\left[\Psi_{q}, 1\right] \subseteq \mathcal{S}_{q}$.

One advantages of polyhedral Lyapunov functions is that they can be computed numerically. Several methods for automated construction of the polyhedral Lyapunov function have been proposed in the literature. Early results include [8], where the Lyapunov function construction was reduced to the design of a balanced polytope satisfying some invariance 
properties. An alternative approach was given by Molchanov and Pyatnitskiy in [19], where algebraic stability conditions based on weighted infinity norms were proposed. A linear programming based method for solving these conditions was given by Polański in [21]. Recently, in [28], Yfoulis and Shorten proposed a numerical approach, called ray-griding, to calculate polyhedral Lyapunov functions based on uniform partitions of the state-space in terms of ray directions. In addition, the polyhedral Lyapunov functions is suitable for control design, which will be explored in the following sections.

\section{Admissible Switching Signals}

Without loss of generality, it is assumed that each subsystem is UUB with decay rate $\beta_{q}$ along with a polyhedral Lyapunov function, $\Psi_{q}(x)$. Denote $\mathcal{P}_{q}=\bar{N}\left[\Psi_{q}, 1\right] \subseteq \mathcal{S}_{q}$, which is a polyhedral C-set and can be described as

$$
\mathcal{P}_{q}=\left\{x \in \mathbb{R}^{n}: F^{q} x \leq \overline{1}\right\},
$$

where $F^{q} \in \mathbb{R}^{m_{q} \times n}, \overline{1}=[1, \cdots, 1]^{T} \in \mathbb{R}^{m_{q}}$ and " $\leq$ " is with respect to componentwise. Then, it holds that

$$
\Psi_{q}(x)=\max _{1 \leq i \leq m_{q}}\left\{f_{i}^{q} x\right\}
$$

where $f_{i}^{q} \in \mathbb{R}^{1 \times n}$ is the $i$-th row of the matrix $F^{q}$ for $i=$ $1, \cdots, m_{q}$.

First, we briefly describe some necessary notations from convex analysis. Given a polyhedral C-set $\mathcal{P}$, let $\operatorname{vert}\left(\mathcal{P}_{q}\right)=$ $\left\{v_{1}, v_{2}, \cdots, v_{N}\right\}$ denote its vertices, and $\operatorname{face}\left(\mathcal{P}_{q}\right)=$ $\left\{F_{1}^{q}, F_{2}^{q}, \cdots, F_{M_{q}}^{q}\right\}$ its facets. The hyperplane that corresponds to the $k$-th facet $F_{k}^{q}$ is defined by $H_{k}^{q}=\left\{x \in \mathbb{R}^{n}\right.$ : $\left.f_{k}^{q} x=1\right\}$, where $f_{k}^{q} \in \mathbb{R}^{1 \times n}$ is the corresponding normal vector of facet $F_{k}^{q}$. The set of vertices of $F_{k}^{q}$ can be found as $\operatorname{vert}\left(F_{k}^{q}\right)=\operatorname{vert}\left(\mathcal{P}_{q}\right) \cap F_{k}^{q}$. Finally, we denote the cone generated by the vertices of $F_{k}^{q}$ by $\operatorname{cone}\left(F_{k}^{q}\right)=\left\{x \in \mathbb{R}^{n}\right.$ : $\left.\sum_{i} \alpha_{i} v_{k_{i}}^{q}, \alpha_{i} \geq 0, v_{k_{i}}^{q} \in \operatorname{vert}\left(F_{k}^{q}\right)\right\}$. The cone $\left(F_{k}^{q}\right)$ has the property that $\forall x \in \operatorname{cone}\left(F_{k}^{q}\right), \Psi(x)=f_{k}^{q} x$.

Next, we characterize a conic partition of the state space based on these polyhedral Lyapunov functions $\Psi_{q}(x)$. Consider any pair of subsystems with modes $q_{1}$ and $q_{2}$, with $q_{1} \neq q_{2} \in Q$, we want to compute the region

$$
\Omega_{q_{1}}^{q_{2}}=\left\{x \in R^{n}: \Psi_{q_{1}}(x) \leq \Psi_{q_{2}}(x)\right\}
$$

For this purpose, we first consider a pair of faces $F_{i_{1}}^{q_{1}}$ and $F_{i_{2}}^{q_{2}}$ of the polyhedral C-sets $\mathcal{P}_{q_{1}}$ and $\mathcal{P}_{q_{2}}$ respectively and consider

$$
C_{q_{1}, i_{1}}^{q_{2}, i_{2}}=\operatorname{cone}\left(F_{i_{1}}^{q_{1}}\right) \cap \operatorname{cone}\left(F_{i_{2}}^{q_{2}}\right)
$$

The set $C_{q_{1}, i_{1}}^{q_{2}, i_{2}}$ is either empty or a polyhedral cone. If $C_{q_{1}, i_{1}}^{q_{2}, i_{2}} \neq \emptyset$, then all the state $x \in C_{q_{1}, i_{1}}^{q_{2}, i_{2}}$ has the property that, $\Psi_{q_{1}}(x)=f_{i_{1}}^{q_{1}} x$ and $\Psi_{q_{2}}(x)=f_{i_{2}}^{q_{2}} x$. Next, we intersect the set $C_{q_{1}, i_{1}}^{q_{2}, i_{2}}$ with the half-space defined by

$$
H F_{q_{1}, i_{1}}^{q_{2}, i_{2}}=\left\{x \in R^{n}:\left(f_{i_{1}}^{q_{1}}-f_{i_{2}}^{q_{2}}\right) x \leq 0\right\}
$$

and get the set $\Omega_{q_{1}, i_{1}}^{q_{2}, i_{2}}=C_{q_{1}, i_{1}}^{q_{2}, i_{2}} \cap H F_{q_{1}, i_{1}}^{q_{2}, i_{2}}$. The reason for specifying the region $\Omega_{q_{1}, i_{1}}^{q_{2}, i_{2}}$ can be clarified by the following lemma [14].

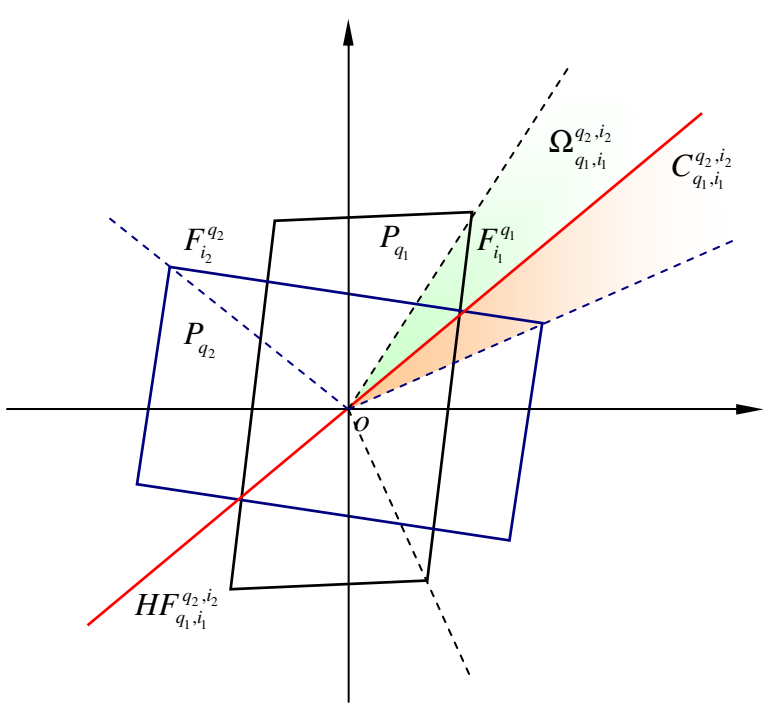

Fig. 1. Illustrations for two polyhedral C-sets $\mathcal{P}_{q_{1}}$ and $\mathcal{P}_{q_{2}}$, their two face $F_{i_{1}}^{q_{1}}$ and $F_{i_{2}}^{q_{2}}$ respectively, the corresponding polyhedral cone $C_{q_{1}, i_{1}}^{q_{2}, i_{2}}=$ $\operatorname{cone}\left(F_{i_{1}}^{q_{1}}\right) \cap \operatorname{cone}\left(F_{i_{2}}^{q_{2}}\right)$, the hyperplane $H_{\left(q_{1}, i_{1}\right)}^{\left(q_{2}, i_{2}\right)}$, and the conic region of $\Omega_{q_{1}, i_{1}}^{q_{2}, i_{2}}$.

Lemma 2: For every $x \in \Omega_{q_{1}, i_{1}}^{q_{2}, i_{2}}$, we have that $\Psi_{q_{1}}(x) \leq$ $\Psi_{q_{2}}(x)$.

Proof: By definition, $\Omega_{q_{1}, i_{1}}^{q_{2}, i_{2}}=C_{q_{1}, i_{1}}^{q_{2}, i_{2}} \cap H F_{q_{1}, i_{1}}^{q_{2}, i_{2}}$, where $C_{q_{1}, i_{1}}^{q_{2}, i_{2}}=\operatorname{cone}\left(F_{i_{1}}^{q_{1}}\right) \cap \operatorname{cone}\left(F_{i_{2}}^{q_{2}}\right)$. The cone $\left(F_{i_{1}}^{q_{1}}\right)$ and $\operatorname{cone}\left(F_{i_{2}}^{q_{2}}\right)$ have the property that $\forall x \in \operatorname{cone}\left(F_{i_{1}}^{q_{1}}\right)$, $\Psi_{q_{1}}(x)=f_{i_{1}}^{q_{1}} x$, and $\forall x \in \operatorname{cone}\left(F_{i_{2}}^{q_{2}}\right), \Psi_{q_{2}}(x)=f_{i_{2}}^{q_{2}} x$. Note that $\forall x \in H F_{q_{1}, i_{1}}^{q_{2}, i_{2}}, f_{i_{1}}^{q_{1}}(x) \leq f_{i_{2}}^{q_{2}}(x)$. Therefore, for all $x \in \Omega_{q_{1}, i_{1}}^{q_{2}, i_{2}}$, we have that $\Psi_{q_{1}}(x) \leq \Psi_{q_{2}}(x)$.

The above notations and the conic region $\Omega_{q_{1}, i_{1}}^{q_{2}, i_{2}}$ are illustrated in Figure 1. Notice that the hyperplane $H_{\left(q_{1}, i_{1}\right)}^{\left(q_{2}, i_{2}\right)}=$ $\left\{x \in R^{n}:\left(f_{i_{2}}^{q_{2}}-f_{i_{1}}^{q_{1}}\right) x=0\right\}$ goes through the origin and the intersection of the faces $F_{i_{1}}^{q_{1}}$ and $F_{i_{2}}^{q_{2}}$. This comes from the fact that $\Psi_{q_{1}}(0)=\Psi_{q_{2}}(0)=0$, and for $x \in$ $F_{i_{1}}^{q_{1}} \cap F_{i_{2}}^{q_{2}} \Rightarrow \Psi_{q_{1}}(x)=\Psi_{q_{2}}(x)=1$. We will show later that this observation simplifies the design procedure for conic partition based switching law.

Based on the above lemma, we have

$$
\Omega_{q_{1}}^{q_{2}}=\bigcup_{i_{1}, i_{2}} \Omega_{q_{1}, i_{1}}^{q_{2}, i_{2}}
$$

where $i_{1}$ and $i_{2}$ go through all the faces' index of $\mathcal{P}_{q_{1}}$ and $\mathcal{P}_{q_{2}}$ respectively. The following corollary holds.

Corollary 1: For every $x \in \Omega_{q_{1}}^{q_{2}}=\bigcup_{i_{1}, i_{2}} \Omega_{q_{1}, i_{1}}^{q_{2}, i_{2}}$, we have that $\Psi_{q_{1}}(x) \leq \Psi_{q_{2}}(x)$.

Because $\Omega_{q_{1}, i_{1}}^{q_{2}, i_{2}}$ is an intersection of a polyhedral cone with a half-space, so it is either an empty set or a polyhedral cone. Hence $\Omega_{q_{1}}^{q_{2}}$ is finite union of polyhedral cones. And due to the fact that $\bigcup_{i_{2}} \operatorname{cone}\left(F_{i_{1}}^{q_{1}}\right)=\bigcup_{i_{2}} \operatorname{cone}\left(F_{i_{2}}^{q_{2}}\right)=\mathbb{R}^{n}$, it is obvious that for $x \notin \Omega_{q_{1}}^{q_{2}}$, we have that $\Psi_{q_{1}}(x) \geq \Psi_{q_{2}}(x)$. Therefore, $\Omega_{q_{1}}^{q_{2}} \cup \Omega_{q_{2}}^{q_{1}}=\mathbb{R}^{n}$.

Now define the following class of switching signals, $\Sigma$, such that for any switching signal $\sigma(t) \in \Sigma$ 
1) $\sigma(t)$ only contains finite number of discontinuities within any finite time interval;

2) If $\sigma(t)=q_{i}$ and $\sigma\left(t^{+}\right)=q_{j}$, then $x(t) \in \Omega_{q_{j}}^{q_{i}}$.

The first condition excludes the occurrence of sliding motions, while the second requirement says that a switching from $q_{i}$ to $q_{j}$ could occur at time $t$ only if $x(t) \in \Omega_{q_{j}}^{q_{i}}$. Notice that arbitrarily fast switching is not excluded. For example, it is possible that " $q_{i}$ to $q_{j}$ then to $q_{k}$ " occurs instantly at $t$ if $x(t) \in \Omega_{q_{j}}^{q_{i}} \cap \Omega_{q_{k}}^{q_{j}}$. It is also clear there are possibly infinite number of switching in a $\sigma(t) \in \Sigma$ (Next section provides an example). The following theorem shows that $\Sigma$ defines a class of admissible switching signals and provides an answer for Problem 1.

Theorem 1: The switched system (2) remains UUB under any switching signal $\sigma(t) \in \Sigma$.

Proof: Define $V(x(t))=\Psi_{\sigma(t)}(x(t))$. For any nonswitching time instant $t, \mathcal{D}^{+} V(x(t))<0$ by assumption of each subsystem is UUB. At switching instant, $V(x(t))$ is decreasing due to Corollary 1. The UUB of the switched system (2) follows from Multiple Lyapunov Theorem [7].

Remark 1: In order to calculate the region $\Omega_{q_{i}}^{q_{j}}$, we simply draw the radii that start from the origin and go through the intersection points of faces of $\mathcal{P}_{q_{i}}$ and $\mathcal{P}_{q_{j}}$. These radii partition the state space into a finite union of conic regions. Notice that along any such radii, $\Psi_{q_{i}}(x)=\Psi_{q_{j}}(x)$, and that within each conic region generated by these radii either $\Psi_{q_{i}}(x) \geq \Psi_{q_{j}}(x)$ or $\Psi_{q_{i}}(x) \leq \Psi_{q_{j}}(x)$ holds. Therefore, $\Omega_{q_{i}}^{q_{j}}$ is just the union of some of these conic regions. To determine whether one of these polyhedral cones is contained in $\Omega_{q_{i}}^{q_{j}}$, one simply checks whether there exists one point in this cone which is on the edge of $\mathcal{P}_{q_{i}}$ but not contained in $\operatorname{int}\left(\mathcal{P}_{q_{j}}\right)$. If such points exist in the cone, then this cone is included into the region $\Omega_{q_{i}}^{q_{j}}$ (from the geometric interpretation of Minkowski function). The region $\Omega_{q_{i}}^{q_{j}}$ is just the union of such cones.

\section{Improved Disturbance AtTEnuAtion Property}

As one of the main motivations to study switched systems, a multi-modal controller can achieve better performance than a single-modal controller [11], an interesting question is whether one can characterize a subclass of admissible switching signals under which (2) achieves an improved disturbance attenuation level. The improvement is in the sense that all possible state trajectories of (2) under the designed switching signals would finally converge to a smaller neighborhood region $(\mathcal{S}$ in Definition 1$)$ of the origin than any single subsystem acts alone. This will be the focus for the rest of the paper.

Problem 2: Given the switched system (2), synthesize switching signals $\sigma(\cdot)$ to assure that the state $x(t)$ is uniformly ultimately bounded into a $\mathrm{C}$-set, which is a subset of all $\mathcal{S}_{q}$.

For this, define a new cone for each $q \in Q$ as

$$
\Omega^{q}=\bigcap_{q_{i} \in Q, q_{i} \neq q} \Omega_{q_{i}}^{q},
$$

which has the property as follows.

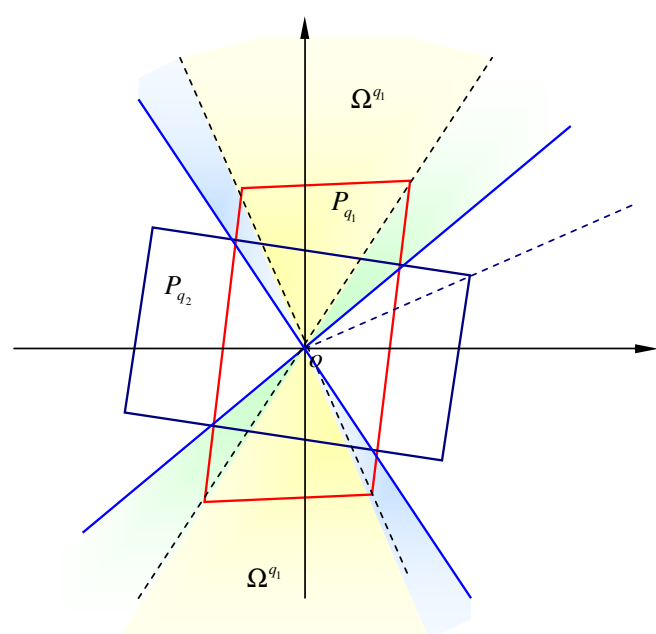

Fig. 2. Illustration for the conic region of $\Omega^{q_{1}}$.

Lemma 3: For every $x \in \Omega^{q}$, we have that $\Psi_{q}(x) \geq$ $\Psi_{q_{i}}(x), \forall q_{i} \in Q$ and $q_{i} \neq q$.

Proof: By definition, $\Omega^{q}=\bigcap_{q_{i} \in Q, q_{i} \neq q} \Omega_{q_{i}}^{q}$. Therefore, $\forall x \in \Omega^{q}=\bigcap_{q_{i} \in Q, q_{i} \neq q} \Omega_{q_{i}}^{q}$, then $x \in \Omega_{q_{i}}^{q}$, for all $q_{i} \in Q$, $q_{i} \neq q$. Note that $\forall x \in \Omega_{q_{i}}^{q}, \Psi_{q_{i}}(x) \leq \Psi_{q}(x)$, which is from the definition of $\Omega_{q_{i}}^{q}$ and Corollary 1. Hence, for every $x \in \Omega^{q}, \Psi_{q}(x) \geq \Psi_{q_{i}}(x), \forall q_{i} \in Q$ and $q_{i} \neq q$.

Some observations about $\Omega^{q}$ are important for the following design procedure. First, in the region of $\Omega^{q}, q \in$ $\arg \max _{q \in Q} \Psi_{q}(x)$. Secondly, $\Omega^{q}$ is finite union of polyhedral cones. Finally, for $x \in \Omega^{q} \cap \Omega^{q^{\prime}}, \Psi_{q}(x)=\Psi_{q^{\prime}}(x)$, and

$$
\bigcup_{q \in Q} \Omega^{q}=\mathbb{R}^{n} .
$$

Therefore, $\Omega^{q}, q \in Q$, provides a conic partition of the state space, based on which a switching law can be defined as

$$
x(t) \in \Omega^{q} \Rightarrow \sigma(t)=q
$$

An illustration of $\Omega^{q}$ is given in Figure 2 .

It can be shown that the switching law defined in (10) can guarantee the UUB for the uncertain switched system in $\bigcap_{q \in Q} \mathcal{P}_{q}$.

Theorem 2: Assume that there is no sliding motion generated by the switching law (10). Then, the uncertain continuous-time switched system (2) is UUB under (10) and state trajectories converge into the polyhedral $\mathrm{C}$-set $\bigcap_{q \in Q} \mathcal{P}_{q}$, which is a subset of any $\mathcal{S}_{q}$.

Proof: Define the function $V(x(t))=$ $\max _{q \in Q} \Psi_{q}(x(t))$. For all $x(t) \notin \bigcap_{q \in Q} \mathcal{P}_{q}$, $V(x(t))=\max _{q \in Q} \Psi_{q}(x(t))>1$. Assume that $x(t) \in \Omega^{q}$ and current mode $q(t)=q$. If no switching occurs at $t$, then there exists $\bar{\tau}>0$ such that $\forall 0<\tau \leq \bar{\tau}, x(t+\tau) \in \Omega^{q}$ and $x(t+\tau) \notin \operatorname{int}\left(\mathcal{P}_{q}\right)$. Then $V(x(t))=\max _{q \in Q} \Psi_{q}(x(t))=\Psi_{q}(x(t))$ and $V(x(t+\tau))=\Psi_{q}(x(t+\tau))$. Then we derive that

$$
\mathcal{D}^{+} V(x(t))=\mathcal{D}^{+} \Psi_{q}(x(t)) \leq-\beta_{q}
$$


Else, if switching occurs at time $t$, then there exists $\bar{\tau}>0$ (due to no-sliding motion assumption) such that $\forall 0<\tau \leq \bar{\tau}$, $x(t+\tau) \in \Omega_{q^{\prime}}$ and $x(t+\tau) \notin \operatorname{int}\left(\mathcal{P}_{q^{\prime}}\right)$. Then $V(x(t))=$ $\max _{q \in Q} \Psi_{q}(x(t))=\Psi_{q}(x(t))=\Psi_{q^{\prime}}(x(t))$ and $V(x(t+$ $\tau))=\Psi_{q^{\prime}}(x(t+\tau))$. Therefore,

$\mathcal{D}^{+} V(x(t))=\lim \sup _{\tau \rightarrow 0^{+}} \frac{\Psi_{q^{\prime}}(x(t+\tau))-\Psi_{q^{\prime}}(x(t))}{\tau} \leq-\beta_{q^{\prime}}$.

Therefore, the uncertain switched system (2) is UUB with respect to the region $\bigcap_{q \in Q} \mathcal{P}_{q}$. Since $\mathcal{P}_{q} \subseteq \mathcal{S}_{q}$, so $\bigcap_{q \in Q} \mathcal{P}_{q}$ is a subset of any $\mathcal{S}_{q}$.

It is interesting to notice that the switching law defined by (10) (when there is no sliding motion occurring) is contained in $\Sigma$ characterized in Section IV, and that the discrete mode is determined by a form of static state feedback.

\section{HANDLING SLIDING Motion}

So far, our arguments are under the assumption that no sliding motion is generated by the switching law (10). However, sliding motions may occur through the proposed conic partition based switching laws. It is also possible that the generated sliding motion causes instability in the closedloop switched systems. Therefore, it is important to explicitly consider sliding motions, especially those sliding motions that may generate divergent trajectories. In this section, we propose an approach to deal with possible unstable sliding motions. In the sequel, it is assumed that an unstable sliding motion occurs between modes $q_{i}$ and $q_{j}$, and the sliding surface lies in the region $\Omega^{q_{i}} \cap \Omega^{q_{j}}$. Actually, it is straightforward to detect the existence of a sliding motion via checking the directions of the vector fields around a given switching surface.

In the following, we will show that it is always possible to get rid of the unstable sliding motions via requesting certain amount of minimum dwell time at the mode $q_{i}$ and $q_{j}$ before the switching. In other words, when switching into the mode $q_{i}$ (or $q_{j}$ ), the switched system has to remain in the mode $q_{i}$ (or $q_{j}$ ) for a time interval with length no less than $\tau_{i}$ (or $\tau_{j}$ ) before the next switching. It is interesting to mention that one may always to pick proper value of $\tau_{i}$ (or $\tau_{j}$ ) such that the UUB preserves under the modified switching law.

To illustrate the idea, consider the following scenario as shown in Figure 3. A state trajectory starts from the region $\Omega^{q_{i}}$, hits the switching surface $\Omega^{q_{i}} \cap \Omega^{q_{j}}$ at time $t_{0}$ and triggers the switching to the mode $q_{j}$ at $t_{0}^{+}$. Then, the mode $q_{j}$ will drive the state back to the region $\Omega^{q_{i}}$ immediately (due to the sliding motion assumption between modes $q_{i}$ and $q_{j}$ ). According to the minimum dwell time requirement, the system remains at the mode $q_{j}$ for at least certain amount of time period, say $\tau_{j}$. If the mode $q_{i}$ is activated again at time $t_{1}$, i.e., $x\left(t_{1}\right) \in \Omega^{q_{i}}$ and $t_{1}-t_{0} \geq \tau_{j}$, which is assumed to drive the state towards region $\Omega^{q_{j}}$ again ${ }^{1}$. Furthermore, the system switches to mode $q_{j}$ again at $t_{2}$ when $x\left(t_{2}\right)$ reach $\Omega^{q_{j}}$ and $t_{2}-t_{1} \geq \tau_{i}$. As this process continuous, the switched system generates zig-zag kind of behavior around

\footnotetext{
${ }^{1}$ This is the worst case for stability consideration.
}

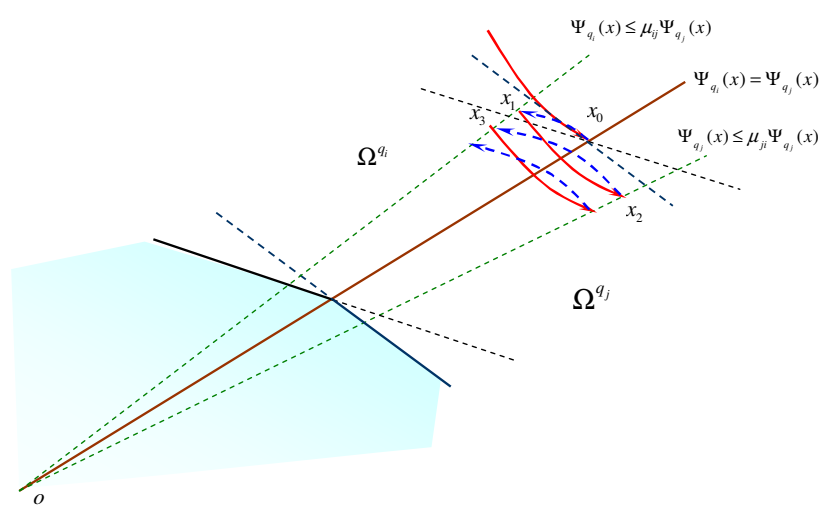

Fig. 3. Hysteresis like switching policy is employed when unstable sliding motions occur between modes $q_{i}$ and $q_{j}$.

the switching surface as shown in Figure 3. Denote the time instants that the switching $q_{i} \rightarrow q_{j}$ occurs as $t_{2 k}$, while $t_{2 k+1}$ as the time instants when $q_{j}$ switches to $q_{j}$.

Several useful properties about the piecewise linear Lyapunov functions $\Psi_{q_{i}}(x)$ and $\Psi_{q_{j}}(x)$ are:

Lemma 4: For $x \in \Omega^{q_{i}} \cap \Omega^{q_{j}}$, it holds that $\Psi_{q_{i}}(x)=$ $\Psi_{q_{j}}(x)$.

Lemma 5: For $x \in \Omega^{q_{i}}, \Psi_{q_{i}}(x) \geq \Psi_{q_{j}}(x)$, but there exists a constant scalar $\mu_{i j} \geq 1$ such that $\Psi_{q_{i}}(x) \leq \mu_{i j} \Psi_{q_{j}}(x)$. $\square$

A possible choice for $\mu_{i j}$ is the largest value among $\Psi_{q_{i}}\left(v_{m}\right), \forall v_{m} \in \operatorname{vert}\left\{\bar{N}\left[\Psi_{q_{j}}, 1\right] \cap \Omega^{q_{i}}\right\}$. This can be verified by exploring the geometric property of the level sets of $\Psi_{q_{i}}(x)$ and $\Psi_{q_{j}}(x)$.

With this, we turn to derive conditions on the determination of $\tau_{i}$ and $\tau_{j}$.

$$
\begin{aligned}
& \Psi_{j}\left(x\left(t_{2}\right)\right) \leq e^{-\beta_{j}\left(t_{2}-t_{1}\right)} \Psi_{j}\left(x\left(t_{1}\right)\right) \\
& \leq e^{-\beta_{j} \tau_{j}} \Psi_{j}\left(x\left(t_{1}\right)\right) \leq e^{-\beta_{j} \tau_{j}} \mu_{j i} \Psi_{i}\left(x\left(t_{1}\right)\right) \\
& \leq e^{-\beta_{j} \tau_{j}} \mu_{j i} e^{-\beta_{i} \tau_{i}} \Psi_{i}\left(x\left(t_{0}\right)\right) \\
&=e^{-\beta_{j} \tau_{j}} \mu_{j i} e^{-\beta_{i} \tau_{i}} \Psi_{j}\left(x\left(t_{0}\right)\right) \\
& \text { If } e^{-\beta_{j} \tau_{j}} \mu_{j i} e^{-\beta_{i} \tau_{i}} \leq 1, \text { then } \Psi_{j}\left(x\left(t_{2}\right)\right) \leq \Psi_{j}\left(x\left(t_{0}\right)\right) . \\
& \Psi_{i}\left(x\left(t_{3}\right)\right) \leq \mu_{i j} \Psi_{j}\left(x\left(t_{3}\right)\right) \leq \mu_{i j} e^{-\beta_{j}\left(t_{3}-t_{2}\right)} \Psi_{j}\left(x\left(t_{2}\right)\right) \\
& \leq \mu_{i j} e^{-\beta_{j} \tau_{j}} \Psi_{j}\left(x\left(t_{2}\right)\right) \\
& \leq \mu_{i j} e^{-\beta_{j} \tau_{j}} \mu_{j i} \Psi_{i}\left(x\left(t_{2}\right)\right) \\
& \leq \mu_{i j} e^{-\beta_{j} \tau_{j}} \mu_{j i} e^{-\beta_{i} \tau_{i}} \Psi_{i}\left(x\left(t_{1}\right)\right)
\end{aligned}
$$

If $\mu_{i j} e^{-\beta_{j} \tau_{j}} \mu_{j i} e^{-\beta_{i} \tau_{i}} \leq 1$, then $\Psi_{i}\left(x\left(t_{3}\right)\right) \leq \Psi_{i}\left(x\left(t_{1}\right)\right)$.

Since $\mu_{i j} \geq 1$, so $\mu_{i j} e^{-\beta_{j} \tau_{j}} \mu_{j i} e^{-\beta_{i} \tau_{i}} \leq 1$ implies $e^{-\beta_{j} \tau_{j}} \mu_{j i} e^{-\beta_{i} \tau_{i}} \leq 1$. Therefore, if

$$
\mu_{i j} e^{-\beta_{j} \tau_{j}} \mu_{j i} e^{-\beta_{i} \tau_{i}} \leq 1
$$

then $\Psi_{i}\left(x\left(t_{2}\right)\right) \leq \Psi_{i}\left(x\left(t_{0}\right)\right)$ and $\Psi_{i}\left(x\left(t_{3}\right)\right) \leq \Psi_{i}\left(x\left(t_{1}\right)\right)$.

Similarly, it can be shown that if

$$
\mu_{i j} e^{-\beta_{j} \tau_{j}} \mu_{j i} e^{-\beta_{i} \tau_{i}} \leq 1
$$


then $\Psi_{j}\left(x\left(t_{2 k}\right)\right) \leq \Psi_{j}\left(x\left(t_{2 k-2}\right)\right)$ and $\Psi_{i}\left(x\left(t_{2 k+1}\right)\right) \leq$ $\Psi_{i}\left(x\left(t_{2 k-1}\right)\right)$, for $k=1,2, \cdots$. This means that at every time the switched system enters (switches into) a certain subsystem, its corresponding Lyapunov function value is smaller than the value at the previous entering time. By the multiple Lyapunov function (MLF) theory [5], [7], it is known that the above condition, together with the condition that the Lyapunov function decreasing its value within each active region, implies the UUB of the switched systems.

Hence, what remains is to pick proper value of $\tau_{i}$ and $\tau_{j}$ such that the inequality

$$
\mu_{i j} e^{-\beta_{j} \tau_{j}} \mu_{j i} e^{-\beta_{i} \tau_{i}} \leq 1
$$

holds.

Notice that $\mu_{i j}$ and $\mu_{i j}$ are finite constants, and that $\beta_{i}$ and $\beta_{j}$ are all positive scalars, so one may always pick $\tau_{i} \geq 0$ and $\tau_{j} \geq 0$ to make (11) holds. One possible choice is

$$
\tau_{i} \geq \frac{\ln \mu_{j i}}{\beta_{i}}, \tau_{j} \geq \frac{\ln \mu_{i j}}{\beta_{j}},
$$

which implies $\mu_{i j} e^{-\beta_{j} \tau_{j}} \leq 1$ and $\mu_{j i} e^{-\beta_{i} \tau_{i}} \leq 1$.

Remark 2: If all the subsystems are LTI systems, then the introduced delayed switching strategy is actually equivalent to hysteresis switching. If sliding motion occur, one may always transfer it into a stable hysteresis switching.

\section{CONCLUDing REMARKS}

In this paper, we investigated the asymptotic disturbance attenuation properties for a class of switched linear systems with parametric uncertainties and exterior disturbances under various switching signals. The main contribution here is the characterization of a class of admissible switching signals under which the switched systems remains UUB. In addition, a subclass of the admissible switching signals is synthesized such that the disturbance attenuation property of the switched system is improved. The improvement is in the sense that state trajectories finally converge into a smaller neighborhood region of the origin than any single subsystem acts alone. The switching law is given as a static state feedback form, and provides a conic partition of the state space. To avoid unstable sliding motions, a modified switching scheme via requesting minimum dwell time is proposed, and a lower bound for the dwell time is estimated. The techniques are based on multiple polyhedral Lyapunov functions.

\section{REFERENCES}

[1] A. Bemporad, G. Ferrari-Trecate, and M. Morari. Observability and controllability of piecewise affine and hybrid systems. IEEE Trans. Automat. Contr., 45(10):1864-1876, 2000.

[2] A. Bemporad and M. Morari. Control of systems integrating logic, dynamics, and constraints. Automatica, 35(3):407-427, 1999.

[3] F. Blanchini. Ultimate boundedness control for uncertain discrete-time systems via set-induced Lyapunov functions. IEEE Trans. Automat. Contr., 39(2):428-433, 1994.
[4] F. Blanchini. Nonquadratic Lyapunov functions for robust control. Automatica, 31(3):451-461, 1995.

[5] M. S. Branicky. Multiple Lyapunov functions and other analysis tools for switched and hybrid systems. IEEE Trans. Automat. Contr., 43(4):475-482, 1998.

[6] M. A. Dahleh and I. Diaz-Bobillo. Control of uncertain systems: A linear programming approach. Prentice Hall, 1994.

[7] R. A. Decarlo, M. S. Branicky, S. Pettersson, and B. Lennartson. Perspectives and results on the stability and stabilizability of hybrid systems. In P. J. Antsaklis, editor, Proceedings of the IEEE: Special issue on hybrid systems, volume 88, pages 1069-1082. IEEE Press, 2000.

[8] K. Drayton and C. Tong. Constructive stability and asymptotic stability of dynamical systems. IEEE Trans. Circuits and Systems, CAS27:1121-1130, 1980.

[9] M. Egerstedt and M. Babaali. On observability and reachability in a class of discrete-time switched linear systems. In Proc. 2005 American Control Conf., pages 1179-1180, 2005.

[10] J. P. Hespanha. Root-mean-square gains of switched linear systems. IEEE Trans. Automat. Contr., 48(11):2040-2045, 2003.

[11] J. P. Hespanha and A. S. Morse. Switching between stabilizing controllers. Automatica, 38(11):1905-1907, 2002.

[12] J.P. Hespanha, D. Liberzon, D. Angeli, and E.D. Sontag. Nonlinear norm-observability notions and stability of switched systems. IEEE Trans. Automat. Contr., 52(2):154-168, 2005.

[13] M. Johansson. Piecewise Linear Control Systems. PhD thesis, Lund Institute of Technology, Sweden, 1999.

[14] X. Koutsoukos and P. Antsaklis. Design of stabilizing switching control laws for discrete and continuous-time linear systems using piecewise-linear Lyapunov functions. Inter. J. Contr., 75(12):932-945, 2002.

[15] D. Liberzon and A. S. Morse. Basic problems in stability and design of switched systems. IEEE Contr. Syst. Magazine, 19(5):59-70, 1999.

[16] H. Lin and P. J. Antsaklis. Synthesis of uniformly ultimate boundedness switching law for discrete-time uncertain switched linear systems. In Proc. 42nd IEEE Conf. Decision Control, pages 4806-4811, 2003.

[17] H. Lin and P. J. Antsaklis. Stability and stabilizability of switched linear systems: A short survey of recent results. In Proc. of 2005 ISIC-MED Joint Conference, pages 24-29, 2005.

[18] A. N. Michel. Recent trends in the stability analysis of hybrid dynamical systems. IEEE Trans. Circuits Syst. I, 46(1):120-134, 1999.

[19] A. P. Molchanov and E. Pyatnitskiy. Criteria of asymptotic stability of differential and difference inclusions encountered in control theory. Systems \& Control Letters, 13:59-64, 1989.

[20] S. Pettersson. Synthesis of switched linear systems. In Proc. 42nd IEEE Conf. Decision Control, pages 5283-5288, 2003.

[21] A. Polański. On absolute stability analysis by polyhedral Lyapunov functions. Automatica, 36(4):573-578, 2000.

[22] Z. Sun and S. S. Ge. Analysis and synthesis of switched linear control systems. Automatica, 41(2):181-195, 2005.

[23] Z. Sun and S. S. Ge. Switched linear systems: Control and design. Springer-Verlag, 2005.

[24] Z. Sun, S. S. Ge, and T. H. Lee. Controllability and reachability criteria for switched linear systems. Automatica, 38(5):775-786, 2002.

[25] X. Xu and P. J. Antsaklis. Optimal control of switched systems based on parameterization of the switching instants. IEEE Trans. Automat. Contr., 49(1):2-16, 2004.

[26] X. Xu and G. Zhai. Practical stability and stabilization of hybrid and switched systems. IEEE Trans. Automat. Contr., 50(11):1897-1903, 2005.

[27] X. Xu, G. Zhai, and S. He. Stabilizability and practical stabilizability of continuous-time switched systems: A unified view. in Proc. of the 2007 American Control Conference, New York, NY, July 2007.

[28] C. Yfoulis and R. Shorten. A numerical technique for stability analysis of linear switched systems. Inter. J. Contr., 77(11):1019-1039, 2004.

[29] G. Zhai, B. Hu, K. Yasuda, and A. N. Michel. Disturbance attenuation properties of time-controlled switched systems. J. Franklin Institute, 338:765-779, 2001. 